\title{
Subjektiver Laufbahnerfolg bei Leistungsschwächeren in einer Berufsausbildung
}

\section{Claudia Hofmann und Kurt Häfeli}

Für leistungsschwächere Jugendliche ist der Berufseinstieg erschwert und gemessen an objektiven Kriterien (Lohn, Position) sind sie meist beruflich weniger erfolgreich. Wie sieht es jedoch mit der subjektiven Einschätzung des eigenen Laufbahnerfolgs zu Beginn der beruflichen Karriere aus und welche Faktoren beeinflussen diese? Im Rahmen einer Studie (2006-2011) wurden 525 Lernende in Grundbildungen mit eidg. Berufsattest aus vier Branchen sowie 183 Anlehrlinge am Ende ihrer Ausbildung befragt. Die Ergebnisse zeigen, dass der subjektive Laufbahnerfolg vor allem mit Persönlichkeits-, Arbeits- und Ausbildungsmerkmalen zusammenhängt, wobei speziell die betriebliche Ausbildungssituation relevant ist. Soziodemographische Merkmale und schulische Herkunft spielen dagegen nur eine geringfügige Rolle.

\section{Ausgangssituation}

Die Ausbildungssituation und der berufliche Einstieg von jungen Erwachsenen mit Leistungsschwächen sind noch wenig untersucht worden (Häfeli \& Schellenberg, 2009). Wie zufrieden sind Jugendliche und junge Erwachsene mit ihrer Ausbildungssituation? Wie verbunden sind sie mit ihrem Beruf? Wie schätzen sie ihre Leistungen in der Berufsfachschule und im Betrieb ein? Wodurch wird der Laufbahnerfolg beeinflusst?

Antworten auf diese Fragen sind nicht nur aus erkenntnistheoretischen Gründen wichtig, die berufliche Integration von Jugendlichen mit Schwächen und Behinderungen ist auch gesellschaftspolitisch relevant. Der Einstieg in die Berufswelt beginnt in Ländern mit einem dualen Berufsbildungssystem deutlich früher als in anderen Ländern. So befindet sich in der Schweiz und in Deutschland mehr als die Hälfte aller 16-18jährigen Jugendlichen in einer Berufsausbildung (OECD, 2011), die im dualen System zum grösseren Anteil im Betrieb stattfindet. Diese Lebensphase kann deshalb als erster Schritt einer beruflichen Laufbahn betrachtet werden. Für die betroffenen Jugend- 
lichen bedeutet dieser Kontextwechsel einerseits eine Stimulation und Bereicherung, andererseits müssen erhebliche Anpassungsleistungen an die neue Situation vollbracht werden (Elfering, Semmer, Tschan, Kälin \& Bucher, 2007; Neuenschwander, Gerber, Frank \& Rottermann, 2012). Der Anteil der Jugendlichen, der den Einstieg in eine Berufsausbildung oder eine weiterführende Schule erfolgreich meistert, ist in der Schweiz vergleichsweise hoch. Jeder vierte oder fünfte Jugendliche schafft dies jedoch nur über den Umweg von Brückenangeboten oder Zwischenlösungen. Zudem sind Wechsel oder Abbrüche innerhalb der beruflichen Ausbildung relativ häufig (Schmid \& Stalder, 2008). Trotz einer breiten Palette von Ausbildungsmöglichkeiten bleibt jeder zehnte junge Erwachsene ohne anerkannte Ausbildung auf der Sekundarstufe II. Vor allem für schulisch schwächere Jugendliche und solche aus problematischen familiären Verhältnissen oder mit Migrationshintergrund ist die berufliche Integration oft mit Problemen verbunden (Häfeli \& Schellenberg, 2009). Leistungsschwächere Jugendliche hatten bis vor kurzem die Möglichkeit, eine Anlehre zu absolvieren. Mit dem neuen Berufsbildungsgesetz (in Kraft seit 1.1.2004) wurde die Anlehre schrittweise aufgehoben und durch die «zweijährige Grundbildung mit eidgenössischem Berufsattest» (EBA) ersetzt. Dies mit dem Ziel, die Arbeitsmarktfähigkeit und die Durchlässigkeit zu weiterführenden Ausbildungen (Bundesamt für Berufsbildung und Technologie [BBT], 2005) und so letztlich auch die Chancen auf eine erfolgreiche berufliche Laufbahn von Leistungsschwächeren zu verbessern.

Die meisten Studien, welche sich mit Laufbahnerfolg ("career success») befassen, wurden in Ländern mit vollschulischen Ausbildungssystemen durchgeführt (Heslin, 2005). Sie sind fokussiert auf «...white, male, well-educated managers or professionals working in large hierarchical organizations.» (Heslin, 2005, S. 127). Studien über junge Erwachsene zu Beginn ihrer Laufbahn sind eher selten. In einer Literaturübersicht konnten Häfeli und Schellenberg (2009) zwar 58 Studien für die Schweiz zusammentragen. Diese betreffen aber primär die erste Schwelle von der obligatorischen Schule in die nächste Ausbildungsstufe und die Ausbildung selber, kaum aber den Übergang in die Erwerbstätigkeit oder in eine weitere Ausbildung. Zudem beschränkte sich die Analyse von Häfeli und Schellenberg auf den objektiven Laufbahnerfolg (Ausbildungsniveau, Status, Einkommen). In einer Längsschnittstudie der Hochschule für Heilpädagogik konnte gezeigt werden, dass junge Erwachsene mit EBA- oder Anlehrabschluss den Einstieg in den Arbeitsmarkt mehrheitlich finden, wenn auch ihre Arbeitslosigkeit etwas höher liegt als im Durchschnitt dieser Altersgruppe (Hofmann \& Kammermann, 2009). Beeinflusst werden der Erwerbsstatus und das Einkommen durch den Ausbildungstypus (Anlehre oder EBA), die Berufsbranche und das Geschlecht (Kammermann, 2010; Kammermann \& Hättich, 2010). Die subjektive Perspektive (Zufriedenheit, berufliche Verbundenheit u.ä.) und mögliche Einflüsse darauf wurden bei der Gruppe der leistungsschwächeren jungen Erwachsenen bisher kaum untersucht. 


\section{Laufbahnerfolg: Definition und Einflussfak- toren}

Laufbahnerfolg («career success») wird von Seibert und Kraimer (2001) definiert: " ....as the accumulated positive work and psychological outcomes resulting from one's work experiences" (zitiert in Ng, Eby, Sorensen \& Feldman, 2005, S. 368). Oft wird dabei zwischen objektivem (oder extrinsischem) und subjektivem (oder intrinsischem) Laufbahnerfolg unterschieden. Indikatoren für objektiven Laufbahnerfolg sind am häufigsten Lohnhöhe und Anzahl beruflicher Aufstiege (Ng et al., 2005). Der zweite, intrinsische Aspekt wird über die subjektive Einschätzung des beruflichen Erfolgs erhoben; die am meisten verwendeten Indikatoren sind Arbeits- oder Berufszufriedenheit.

Diese häufig verwendeten Kriterien und deren Operationalisierung werden allerdings immer wieder als zu eng und eingeschränkt kritisiert. So fordert Heslin (2005), dass die unterschiedlichen Kontexte, in welchen Individuen ihren beruflichen Erfolg einschätzen, mitberücksichtigt werden. Für den hier untersuchten Kontext von schwächeren Jugendlichen am Anfang ihrer Berufslaufbahn könnte dies beispielsweise heissen, dass sich die subjektive Leistungeinschätzung auf die beiden Ausbildungsorte Betrieb und Berufsschule beziehen muss. Auch dürften am Ende einer Berufsausbildung nicht nur die Ausbildungszufriedenheit sondern auch das Ausmass beruflicher Verbundenheit wichtige Indikatoren für den subjektiven Laufbahnerfolg sein.

$\mathrm{Ng}$ et al. (2005) unterscheiden in ihrer Metaanalyse vier Einflussbereiche für den subjektiven und den objektiven Laufbahnerfolg:

- Sozio-demographische Faktoren umfassen den demographischen und sozialen Hintergrund einer Person (Ethnie, sozioökonomischer Hintergrund, Gender, Zivilstand, Alter etc.).

- Humankapital bezieht sich auf den individuellen Erfahrungshintergrund wie absolvierte Ausbildung, Arbeitserfahrung etc.

- Stabile Persönlichkeitsmerkmale umfassen individuelle Dispositionen wie Persönlichkeit («big five»), proaktive Haltung, Kontrollbewusstsein, kognitive Fähigkeiten etc.

- «Organizational sponsorship» bezieht sich darauf, inwieweit ein Betrieb oder eine Organisation Unterstützung bei der Laufbahnentwicklung anbietet (Vorgesetzten-Unterstützung, betriebliche Ausbildungsmöglichkeiten, organisatorische Ressourcen etc.).

Kritisch anzumerken ist, dass der Einflussbereich "Organizational sponsorship» etwas eng und zu stark auf den beruflichen Aufstieg fokussiert scheint. In einer Metaanalyse zeigen Humphrey, Nahrgang und Morgeson (2007) die Einflüsse von Merkmalen der Arbeitsgestaltung auf eine Reihe von "Work Outcomes» auf. Sie erweitern die «klassischen» arbeitsmotivationalen Dimensionen von Hackman und Oldham wie Autonomie, Vielfalt, Bedeutsamkeit oder Ganzheitlichkeit, indem sie soziale und kontextuelle Faktoren anfügen (Hackman \& 
Oldham, 1976; Humphrey et al., 2007).

Die Einflussbereiche wirken sich unterschiedlich auf den objektiven und subjektiven Laufbahnerfolg aus. Ng et al. (2005) zeigen in ihrer Metananalyse, dass sich beim objektiven Indikator «Salär» vor allem das Humankapital (durchschnittliche nach Stichprobengrösse korrigierte Korrelation: $r_{\mathrm{c}}$ (korrigiert) $=.21)$ und sozio-demographische Variablen $\left(\mathrm{r}_{\mathrm{c}}=.20\right)$ auswirken; beim subjektiven Indikator «career satisfaction" sind es vor allem Variablen des «Organizational sponsorship" $\left(\mathrm{r}_{\mathrm{c}}=.31\right)$ und stabile Persönlichkeitsmerkmale $\left(\mathrm{r}_{\mathrm{c}}=.24\right)$, welche höher korrelieren.

Obwohl sich die Forschung sowohl mit subjektivem wie auch mit objektivem Laufbahnerfolg beschäftigt, befassten sich deutlich mehr empirische Studien mit objektiven Kriterien, sodass gemäss den Einschätzungen von Heslin (2005) und Ng et al. (2005) bei der subjektiven Seite stärkerer Forschungsbedarf besteht. Subjektiver Laufbahnerfolg und die Frage, wodurch er beeinflusst wird, soll denn auch bei unseren Analysen im Zentrum stehen.

Im Folgenden werden dazu einige wichtige Ergebnisse aus der angelsächsischen Literatur ergänzt durch Studien aus dem deutschsprachigen Berufsbildungskontext referiert. Dabei übernehmen wir die Gliederung der Einflussbereiche von $\mathrm{Ng}$ et al. (2005).

\section{Der Einfluss von sozio-demographischen Faktoren}

Wie erwähnt sind diese Faktoren in der Metanalyse von Ng et al. (2005) zwar von erheblicher Bedeutung für den objektiven Laufbahnerfolg, aber von geringer Bedeutung für die subjektive Einschätzung der Situation (wie berufliche Zufriedenheit). Auch für unsere Zielgruppe hat sich gezeigt, dass die sozioökonomische Herkunft und der kulturelle Hintergrund der Jugendlichen die Positionierung und das Niveau der Ausbildung beeinflussen (Häfeli \& Schellenberg, 2009). Für den subjektiven Laufbahnerfolg ist jedoch anzunehmen, dass diese Merkmale keine wesentliche oder möglicherweise eine zwiespältige Rolle spielen: Jugendliche aus benachteiligten Verhältnissen, welche ihre Ansprüche senken mussten («Aspirationsabkühlung»), um überhaupt noch eine Lehrstelle zu finden (Becker \& Hecken, 2008; Herzog, Neuenschwander \& Wannack, 2006) könnten in der Folge mit ihrer Ausbildungssituation unzufriedener sein (Müller, 2009). Dasselbe trifft möglicherweise aber auch für Jugendliche aus privilegierteren Verhältnissen zu, da sie verglichen mit ihrer sozialen Referenzgruppe einen «Abstieg» erleben.

\section{Humankapital: Der Einfluss der schulischen Herkunft}

Auch diese Faktoren haben sich in der Metanalyse von Ng et al. (2005) als wenig einflussreich für die berufliche Zufriedenheit herausgestellt. In unserem Kontext könnte die bisherige schulische Laufbahn allenfalls einen Einfluss ausüben, allerdings sind auch hier wieder zwei Szenarien denkbar: Jugendliche, welche die obligatorische Schulzeit in separierten Spezialklassen oder in einer 
Sonderschule absolvieren, sind im Vergleich mit Lernenden aus Regelklassen in der Ausbildung stärker gefordert, teilweise auch überfordert (Hofmann \& Kammermann, 2008). Andererseits sind sie bei der Suche nach einem Ausbildungsplatz auf Sekundarstufe II mit grösseren Schwierigkeiten konfrontiert als Jugendliche aus Regelklassen (Häfeli \& Schellenberg, 2009). Dies könnte dazu beitragen, dass sie die Ausbildung eher als Chance interpretieren, diese motivierter angehen und vorhandene Defizite zu kompensieren versuchen, während Jugendliche mit besseren schulischen Voraussetzungen ihren Ausbildungsweg möglicherweise eher als Misserfolg werten.

\section{Der Einfluss von stabilen Persönlichkeitsmerkmalen}

In der Übersicht von $\mathrm{Ng}$ et al. (2005) zeigen Persönlichkeitsmerkmale wie Extraversion, proaktives Verhalten, Kontrollüberzeugung oder tiefe Werte bei Neurotizismus eine positive Beziehung zur Laufbahnzufriedenheit. Im deutschsprachigen Berufsbildungskontext konnte gezeigt werden, dass sich ein hoher Selbstwert positiv auf die beruflichen Entwicklung auswirkt (Herzog et al., 2006). Der Selbstwert, das Durchsetzungsvermögen, die berufliche Identifikation und die Passung zwischen Beruf und eigenen Fähigkeiten sowie Interessen beeinflussen gemäss Analysen von Neuenschwander, Frey und Gasser (2007) die Arbeitszufriedenheit positiv. Weiter erwiesen sich die ausbildungsbezogene Motivation, beruflicher Zukunftsoptimismus und die Realisierung des Wunschberufes in der ULME-Studie als wichtige Einflussfaktoren für die Ausbildungszufriedenheit (Lehmann \& Seeber, 2007; Neuenschwander et al., 2007).

\section{Der Einfluss von Arbeits- und Ausbildungsbedingungen}

Institutionelle Massnahmen wie Unterstützung durch Vorgesetzte, vielfältige Ausbildungsmöglichkeiten und Karriereförderung üben gemäss der Metaanalyse von $\mathrm{Ng}$ et al. (2005) den stärksten Einfluss auf die Laufbahnzufriedenheit aus. Humphrey et al. (2007) ergänzten zu den motivationalen Arbeitsmerkmalen soziale und kontextuelle Aspekte: Die motivationalen Aspekte (Autonomie, Vielfalt, Komplexität der Arbeit etc.) zeigen zwar die stärksten Zusammenhänge zu verschiedenen Kriterien des Laufbahn- und Arbeitserfolgs, so etwa zu subjektiver Leistungseinschätzung, beruflicher Identifikation und Arbeitszufriedenheit. Aber auch die sozialen Merkmale (Feedback von Anderen, soziale Unterstützung und gegenseitige Abhängigkeit) beeinflussen verschiedene Indikatoren des subjektiven Laufbahnerfolgs. Begrenzter sind die Aussagen zu kontextuellen Merkmalen (physische Anforderungen, Arbeitsbedingungen), da hier nur wenige Studien vorliegen.

Es ist davon auszugehen, dass sich Arbeits- und Ausbildungsmerkmale wie herausfordernde und abwechslungsreiche Arbeit, kompetente Ausbildungspersonen und ein gutes Klima ebenfalls positiv auf die jungen Erwachsenen auswirken. Dies zeigen sowohl die TREE-Studie (Stalder, 2003) bei Jugendlichen während der Ausbildung, als auch die AEQUAS-Studie bei jungen 
Erwachsenen im Übergang von der Berufsausbildung ins Erwerbsleben (Kälin et al., 2000). Da sich die Berufsausbildung im Lernkontext von Betrieb und Berufsfachschule abspielt, ist es notwendig, diese Lernorte gesondert zu berück-sichtigen. Zusätzlich ist in unserer Studie der Wechsel des Ausbildungssystems zu berücksichtigen (Anlehre - EBA-Ausbildung): Erwünscht wären hier natürlich positivere Einschätzungen des subjektiven Laufbahnerfolgs, denkbar ist allerdings auch, dass die erhöhten Anforderungen zu kritischeren Einschätzungen führen.

\section{Modell und Fragestellungen}

In Abbildung 1 sind auf der Grundlage der Einflussbereiche von $\mathrm{Ng}$ et al. (2005) und Humphrey et al. (2007) und abgeleitet aus der obigen Literaturübersicht in einem Modell eine Reihe möglicher Einflussfaktoren aufgeführt. Diese betreffen zum einen die Herkunft und das Humankapital der jungen Erwachsenen; zum andern dürften aber auch individuelle Persönlichkeitsmerkmale und die Ausbildungssituation in Berufsfachschule und Betrieb eine Rolle spielen.

\section{Abbildung 1: Einflussfaktoren auf subjektiven Laufbahnerfolg (in Anlehnung an} $\mathrm{Ng}$ et al., 2005)

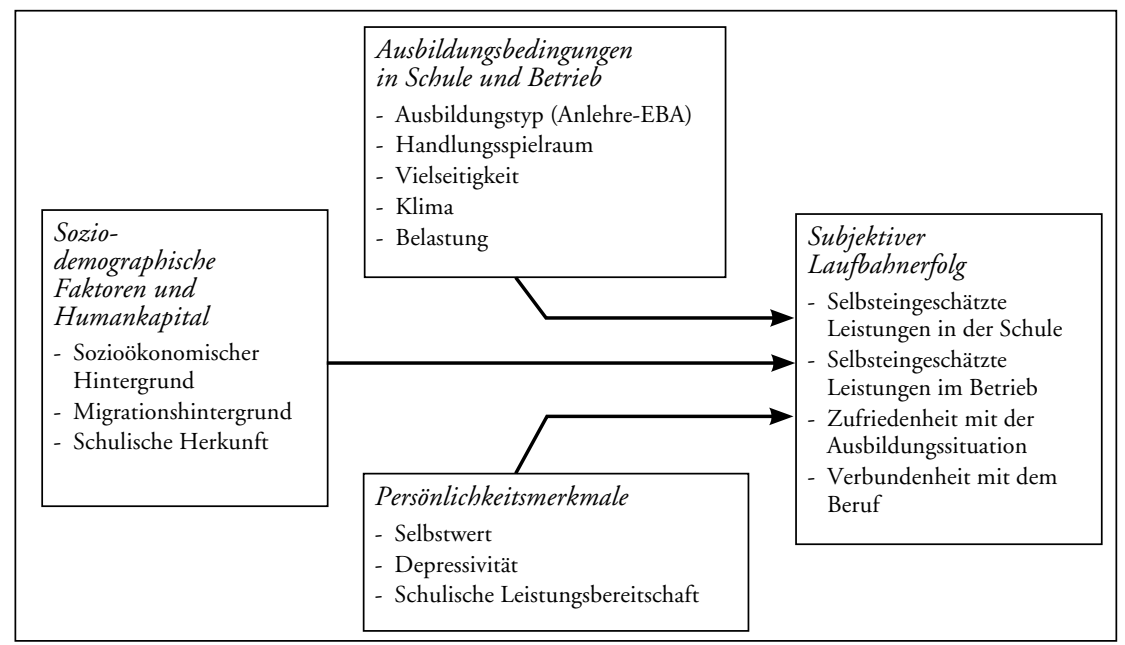

\section{Fragestell lungen}

Ausgehend von einigen zentralen Erkenntnissen aus den Literaturübersichten von $\mathrm{Ng}$ et al. (2005) und Humphrey et al. (2007) soll in unserer Studie der Frage nachgegangen werden, ob diese Befunde auf die Zielgruppe von jungen Erwachsenen mit Leistungsschwächen im schweizerischen Berufsbildungskontext übertragbar sind: 
1) Wird der subjektive Laufbahnerfolg von Lernenden am Ende ihrer Ausbildung stärker durch stabile Persönlichkeitsmerkmale sowie Arbeits- und Ausbildungsmerkmale beeinflusst und weniger durch soziodemographische Merkmale und das Humankapital?

2) Unterscheiden sich Merkmale des betrieblichen und des schulischen Lernorts in ihrem Einfluss auf ausgewählte Kriterien des subjektiven Laufbahnerfolgs?

3) Spielen je nach untersuchtem Kriterium des subjektiven Laufbahnerfolgs unterschiedliche Einflussfaktoren eine Rolle?

\section{Methoden}

\section{Forschungsdesign}

In einem Forschungsprojekt der Hochschule für Heilpädagogik Zürich (HfH) in Zusammenarbeit mit dem Service de la Recherche en Education (SRED) in Genf wurden die Umsetzung und die Auswirkungen der einleitend erwähnten Ausbildungsreform in den Branchen Detailhandel und Gastgewerbe untersucht (2006-2009). Die HfH hat diese Studie in einem Nachfolgeprojekt auf die zweijährigen Grundbildungen in den Branchen Hauswirtschaft und Schreinerei ausgeweitet (2009-2011).

\section{St ichprobe}

In einer ersten Staffel der Erhebungen wurden die letzten Anlehrlinge (2006, $\mathrm{N}=183$ ) sowie die ersten EBA-Lernenden (2007, $\mathrm{N}=319)$ in den Branchen Detailhandel und Gastronomie befragt, wobei die Kantone mit den häufigsten Ausbildungsverhältnissen und drei Sprachregionen berücksichtigt wurden (sieben Deutschschweizer Kantone, drei aus der französischen Schweiz und der Kanton Tessin). Die Lernenden wurden rund zwei Monate vor Ende der zweijährigen Ausbildung schriftlich in ihren Berufsfachschulklassen befragt. Im Zentrum standen Fragen zur bisherigen Schul- und Berufslaufbahn, zur Ausbildungssituation und zu beruflichen Zukunftsperspektiven. Die Studie wurde 2009 in einer zweiten Staffel auf die Branchen Schreinerei und Hauswirtschaft ausgeweitet, wobei dabei nur die Deutschschweiz und die EBA-Ausbildung einbezogen wurden $(\mathrm{N}=206)$.

Die Lernenden verteilten sich wie folgt auf die vier Branchen: $40.8 \%$ absolvierten eine Ausbildung im Detailhandel (14.7\% Anlehre, 26.1\% EBA-Ausbildung), $30.1 \%$ in der Gastwirtschaft (11.2\% Anlehre, 18.9\% EBA-Ausbildung), $15 \%$ in der Hauswirtschaft (alle EBA-Ausbildung) und $14.1 \%$ in der Schreinerei (alle EBA-Ausbildung). Durchschnittlich waren die befragten Lernenden 19.8 Jahre alt ( $\mathrm{SD}=2.3$ Jahre).

Die folgende Tabelle 1 gibt einen Überblick über zusätzliche ausgewählte Merkmale der Stichprobe, die für die weiteren Analysen relevant sind: 
Tabelle 1: Sozioökonomischer Status, Migrationshintergrund, Geschlecht und schulische Herkunft

Beschreibung

Sozioökonomischer Status Index der Familie (ISEI, tiefer Wert=tiefer Status)

Geschlecht

- weiblich $(=1)$

- männlich (=2)

Migrationshintergrund

- selber \& Mutter \& Vater in der Schweiz geboren (=1)

- selber in der Schweiz \& Mutter oder Vater im Ausland geboren (=2)

- selber in der Schweiz \& beide Elternteile im Ausland geboren $(=3)$

- selber \& Eltern im Ausland geboren, > 7 Jahre in der Schweiz (=4)

- selber \& Eltern im Ausland geboren $\leq 7$ Jahre in der Schweiz (=5)

Schulische Herkunft vor Ausbildungsbeginn

- Hauptsächlich (fünf oder mehr Jahre) Regelklassen (=1)
Häufigkeiten/M/SD N

$\mathrm{M}=37.9 \quad 650$

$\mathrm{SD}=14.4$

$436(61.8 \%) \quad 706$

$270(38.2 \%)$

$283(41.4 \%)$

683

$81(11.9 \%)$

$75(11.0 \%)$

$178(26.1 \%)$

$66(9.7 \%)$

$503(73.3 \%)$

686

Die fehlenden Werte sind auf unklare bzw. nicht eindeutige Angaben der Befragten zurückzuführen. Die Ergebnisse der Analysen basieren deshalb auf einer reduzierten Stichprobe.

\section{Erhebungsinstrumente}

Der Fragebogen der Ersterhebungen setzt sich aus im Rahmen des Forschungsprojekts entwickelten, sowie von der Längsschnittstudie TREE (Stalder, Meyer \& Hupka-Brunner, 2011) übernommenen Items zusammen (Kammermann, Amos, Hofmann \& Hättich, 2009), wobei sich diese auf bestehende Konzepte und Operationalisierungen stützen (weitere Kennwerte vgl. Tabelle 2):

\section{Persönlichkeitsmerkmale}

- Schulische Leistungsbereitschaft (z.B. «Wenn ich lerne, gebe ich mein Bestes.»), Fünferantwortskala von «stimmt überhaupt nicht» bis «stimmt genau» (Moser, 1997; Moser, Ramseier, Keller \& Huber, 1997).

- Selbstwert (z.B. «Insgesamt bin ich mit mir selbst zufrieden.») und Depressivität (z.B. «Ich fühle mich manchmal richtig wertlos.»), Fünferantwortskala von «trifft gar nicht zu» bis «trifft genau zu» (Rosenberg, 1979).

\section{Ausbildungsbedingungen in der Schule und im Betrieb}

- Klima im Lehrbetrieb (z.B. «Mein Lehrbetrieb ist ein Ort, wo ich gerne bin.»), Viererantwortskala von «stimmt überhaupt nicht» bis «stimmt genau» (Neuenschwander, 1998). 
- Handlungsspielraum im Lehrbetrieb (z.B. «Ich kann meine Arbeit selbständig einteilen.»), Vielseitigkeit im Lehrbetrieb (z.B. «Meine Arbeit ist abwechslungsreich.») und Belastung im Lehrbetrieb (z.B. "Der Zeitdruck an der Arbeit ist gross.»), Fünferantwortskala von «sehr selten/nie» bis «sehr oft/immer» (Prümper, Hartmannsgruber \& Frese, 1995; Semmer, Zapf \& Dunckel, 1999).

- Klima in der Berufsfachschule (z.B. «Die Schule ist ein Ort, wo ich gerne bin.»), Viererantwortskala von «stimmt überhaupt nicht» bis «stimmt genau» (Neuenschwander, 1998).

- Handlungsspielraum in der Berufsfachschule (z.B. «Ich kann selber bestimmen, auf welche Art und Weise ich meine Arbeit im Unterricht erledige.»), Vielseitigkeit in der Berufsfachschule («Im Unterricht kann ich viel dazu lernen.») und Belastung in der Berufsfachschule ("Der Unterricht geht so schnell weiter, dass ich Schwierigkeiten habe mitzukommen.»). Fünferantwortskala von «sehr selten/nie» bis «sehr oft/immer» (Prümper et al., 1995; Semmer et al., 1999).

\section{Subjektiver Laufbahnerfolg}

- Zufriedenheit mit der Ausbildungssituation (1 Item, «Wie zufrieden sind Sie ganz allgemein mit Ihrer jetzigen Ausbildung?» Sieben-Punkt-Antwortskala von «ausserordentlich unzufrieden» bis «ausserordentlich zufrieden» (Baillod, 1992).

- Verbundenheit mit dem Beruf (z.B. «Ich bin stolz auf den Beruf, den ich lerne.»), Viererantwortskala von «stimmt überhaupt nicht» bis «stimmt genau» (Neuenschwander, 1998).

- Selbsteingeschätzte Leistungen in der Berufsfachschule (allgemein bildender und Fachkundeunterricht) und im Lehrbetrieb (z.B. «Wie schätzen Sie Ihre Leistungen in der praktischen Arbeit im Betrieb ein?»), Fünferantwortskala von «schlecht» bis «gut» (Kammermann, Hofmann \& Hättich, 2009)

Die Reliabilitätswerte der verwendeten Skalen (Cronbach- $\alpha$, vgl. Tabelle 2) sind mehrheitlich zufriedenstellend bis hoch. 
Tabelle 2: Kennwerte zu den Variablen Persönlichkeitsmerkmale, Ausbildungsbedingungen und subjektiver Laufbahnerfolg

Beschreibung

Persönlichkeitsmerkmale

Selbstwert

Depressivität

Schulische Leistungsbereitsschaft

Ausbildungsbedingungen

Ausbildungstypus (Anlehre - EBA)

Klima im Lehrbetrieb

Klima in der Berufsfachschule

Belastung im Lehrbetrieb

Belastung in der Berufsfachschule

Handlungsspielraum Lehrbetrieb

Handlungsspielraum Schule

Vielseitigkeit Lehrbetrieb

Vielseitigkeit Schule

Subjektiver Laufbahnerfolg

Selbsteingeschätzte Leistungen in der Schule

Selbsteingeschätzte Leistungen im Betrieb

Zufriedenheit mit der Ausbildung

Verbundenheit mit dem Beruf

Anzahl Items Cronbach- $\alpha \quad \mathrm{N} \quad \mathrm{M} \quad$ SD

$\begin{array}{lllll}4 & 0.72 & 695 & 3.99 & 0.64 \\ 4 & 0.76 & 699 & 2.48 & 0.92 \\ 5 & 0.87 & 699 & 3.71 & 0.74\end{array}$

$\begin{array}{lllll}3 & 0.84 & 698 & 2.90 & 0.85\end{array}$

$\begin{array}{lllll}3 & 0.78 & 702 & 2.88 & 0.75\end{array}$

$\begin{array}{lllll}5 & 0.72 & 698 & 2.65 & 0.74\end{array}$

$\begin{array}{lllll}5 & 0.80 & 701 & 2.23 & 0.79\end{array}$

$\begin{array}{lllll}3 & 0.70 & 702 & 3.33 & 0.92\end{array}$

$\begin{array}{lllll}3 & 0.64 & 705 & 2.86 & 0.84\end{array}$

$\begin{array}{lllll}3 & 0.75 & 704 & 3.89 & 0.78\end{array}$

$\begin{array}{lllll}3 & 0.62 & 703 & 3.70 & 0.72\end{array}$

$\begin{array}{lcccc}2 & 0.78 & 706 & 3.82 & 0.75 \\ 1 & - & 697 & 4.18 & 0.79 \\ 1 & - & 703 & 5.32 & 1.24 \\ 4 & 0.86 & 686 & 3.11 & 0.73\end{array}$

\section{Auswertungsmethoden}

Mittels linearer Regressionsanalysen wurde untersucht, wie die verschiedenen unabhängigen Variablen mit den Indikatoren für subjektiven Laufbahnerfolg (selbsteingeschätzte Leistungen in Schule und Betrieb, Zufriedenheit mit der Ausbildungssituation, Verbundenheit mit dem Beruf) zusammenhängen. Teilweise erwiesen sich die bivariaten Korrelationen zwischen den unabhängigen Variablen als recht hoch. ${ }^{1}$ Die Multicollinearitätsdiagnostik zeigt jedoch beim VIF (Variance Inflation Factor) keine bedenklichen Werte (Field, 2009; Myers, 1990). Einleitend wird die Korrelationsmatrix aller 16 unabhängigen mit den vier abhängigen Variablen präsentiert (Tab.3). 
Tabelle 3: Interkorrelationen (nach Pearson) zwischen den unabhängigen Variablen und den abhängigen Variablen (selbsteingeschätzte Leistungen, Zufriedenheit, berufliche Verbundenheit)

\begin{tabular}{|c|c|c|c|c|}
\hline & $\begin{array}{l}\text { Selbsteing. } \\
\text { Leistungen } \\
\text { Schule } \\
(\mathrm{N}=583)\end{array}$ & $\begin{array}{l}\text { Selbsteing. } \\
\text { Leistungen } \\
\text { Betrieb } \\
(\mathrm{N}=574)\end{array}$ & $\begin{array}{c}\text { Zufriedenheit } \\
\text { Ausbildung } \\
(\mathrm{N}=580)\end{array}$ & $\begin{array}{c}\text { Verbundenheit } \\
\text { mit Beruf } \\
(\mathrm{N}=570)\end{array}$ \\
\hline Sozioökonomischer Status & -.05 & $-.12^{* *}$ & -.01 & $-.10^{* *}$ \\
\hline Migrationshintergrund & -.01 & $.16^{* * *}$ & .02 & .06 \\
\hline Geschlecht & $-.09^{*}$ & $-.10^{* *}$ & .00 & .04 \\
\hline Schulische Herkunft & -.04 & .02 & $.12^{* *}$ & $.12^{* *}$ \\
\hline Selbstwert & $.27^{* * *}$ & $.37^{* * *}$ & $.24^{* * *}$ & $.26^{* * *}$ \\
\hline Depressivität & $-.18^{* * *}$ & $-.20^{* * *}$ & $-.09^{*}$ & -.07 \\
\hline Schulische Leistungsbereitschaft & $.42^{* * *}$ & $.20^{* * *}$ & $.30^{* * *}$ & $.32^{* * *}$ \\
\hline Vielseitigkeit Betrieb & $.16^{* * *}$ & $.34^{* * *}$ & $.36^{* * *}$ & $.43^{* * *}$ \\
\hline Handlungsspielraum Betrieb & .05 & $.36^{* * *}$ & $.23^{* * *}$ & $.34^{* * *}$ \\
\hline Klima Betrieb & $.11^{* *}$ & $.26^{* * *}$ & $.39^{* * *}$ & $.43^{* * *}$ \\
\hline Belastung Betrieb & $-.08^{*}$ & .01 & $-.11^{* *}$ & -.01 \\
\hline Vielseitigkeit Schule & $.32^{* * *}$ & $.08^{*}$ & $.33^{* * *}$ & $.23^{* * *}$ \\
\hline Handlungsspielraum Schule & $.20^{* * *}$ & .05 & $.15^{* * *}$ & $.20^{* * *}$ \\
\hline Klima Schule & $.35^{* * *}$ & .03 & $.20^{* * *}$ & $.20^{* * *}$ \\
\hline Belastung Schule & $-.31^{* * *}$ & -.01 & $.09^{*}$ & .04 \\
\hline Ausbildungstyp & -.02 & $-.15^{* * *}$ & $.09^{*}$ & $-.11^{* *}$ \\
\hline Selbsteing. Leistungen Schule & - & & & \\
\hline Selbsteing. Leistungen Betrieb & $.29^{* * *}$ & - & & \\
\hline Zufriedenheit Ausbildung & $.18^{* * *}$ & $.20^{* * *}$ & - & \\
\hline
\end{tabular}

Anmerkungen. ${ }^{* * *}=\mathrm{p}<.001,{ }^{* *}=\mathrm{p}<.01,{ }^{*}=\mathrm{p}<.05$

\section{Ergebnisse}

Im Folgenden wird nun analysiert, welche unabhängigen Variablen bei den verschiedenen Dimensionen des beruflichen Laufbahnerfolgs eine Rolle spielen. Die gesamte aufgeklärte Varianz beträgt, je nach abhängiger Variable, zwischen $28 \%$ bis $32 \%$. 
Tabelle 4: Regressionsanalyse zur Vorhersage der selbsteingeschätzten schulischen und betrieblichen Leistungen

\begin{tabular}{|c|c|c|c|c|c|c|}
\hline & \multicolumn{3}{|c|}{$\begin{array}{c}\text { Selbsteingeschätzte } \\
\text { schulische Leistungen } \\
(\mathrm{N}=583)\end{array}$} & \multicolumn{3}{|c|}{$\begin{array}{l}\text { Selbsteingeschätzte } \\
\text { betriebliche Leistungen } \\
\qquad(\mathrm{N}=574)\end{array}$} \\
\hline & B & $\mathrm{SE}(\mathrm{B})$ & $\beta$ & B & $\mathrm{SE}(\mathrm{B})$ & $\beta$ \\
\hline Sozioökonomischer Status & .00 & .00 & -.04 & .00 & .00 & -.05 \\
\hline Migrationshintergrund & -.01 & .02 & -.01 & .06 & .02 & $.11^{* *}$ \\
\hline Geschlecht & -.07 & .06 & -.04 & -.19 & .06 & $-.11^{* *}$ \\
\hline Schulische Herkunft & -.11 & .06 & -.06 & .02 & .07 & .01 \\
\hline Selbstwert & .16 & .05 & $.14^{* *}$ & .28 & .05 & $.22^{* * *}$ \\
\hline Depressivität & -.03 & .03 & -.04 & -.10 & .04 & $-.11^{* *}$ \\
\hline Schulische Leistungsbereit. & .28 & .05 & $.28^{* * *}$ & .11 & .05 & $.10^{*}$ \\
\hline Vielseitigkeit Betrieb & -.01 & .05 & -.01 & .11 & .05 & $.11^{*}$ \\
\hline Handlungsspielraum Betrieb & .01 & .04 & .01 & .20 & .04 & $.23^{* * *}$ \\
\hline Klima Betrieb & -.01 & .04 & -.01 & -.01 & .04 & -.01 \\
\hline Belastung Betrieb & .03 & .04 & .03 & -.01 & .04 & -.01 \\
\hline Vielseitigkeit Schule & .02 & .05 & .02 & -.01 & .06 & -.01 \\
\hline Handlungsspielraum Schule & .05 & .03 & .05 & -.03 & .04 & -.03 \\
\hline Klima Schule & .14 & .04 & $.14^{* *}$ & -.08 & .05 & -.07 \\
\hline Belastung Schule & -.25 & .04 & $-.26^{* * *}$ & .01 & .04 & .01 \\
\hline
\end{tabular}

Anmerkungen: Selbsteingeschätzte Leistungen Schule $\mathrm{R}^{2}=.32, \mathrm{~F}=16.33(\mathrm{df}=16, \mathrm{p}<0.001)$

Selbsteingeschätzte Leistungen Betrieb $\mathrm{R}^{2}=.28, \mathrm{~F}=13.52(\mathrm{df}=16, \mathrm{p}<0.001)$

${ }^{* * *}=\mathrm{p}<.001,{ }^{* *}=\mathrm{p}<.01,{ }^{*}=\mathrm{p}<.05$

\section{Unabhängige Variablengruppen und subjektiver Laufbahnerfolg}

Als erstes werden die Ergebnisse gegliedert nach Variablengruppen präsentiert (vgl. Tabellen 4 und 5) - und damit untersucht, wie Persönlichkeits-, Arbeits-/ Ausbildungsmerkmale, soziodemographische Merkmale und Humankapital mit subjektivem Laufbahnerfolg zusammenhängen (Fragestellung 1).

Wie erwartet zeigen sich nur vereinzelte Zusammenhänge bei den sozio-demographischen Variablen: Jugendliche mit Migrationshintergrund beurteilen die eigenen praktischen Leistungen im Betrieb günstiger als Jugendliche ohne Migrationshintergrund. Deutlich ist der Zusammenhang zwischen den Selbsteinschätzungen und der Geschlechtszugehörigkeit: Männliche Lernende schätzen sich bei den betrieblichen Leistungen tiefer ein als ihre weiblichen Kolleginnen. Schliesslich zeigt sich: Je höher der sozioökonomische Status, desto tiefer ist die berufliche Verbundenheit. 
Tabelle 5: Regressionsanalysen zur Vorhersage der Ausbildungszufriedenheit und der Verbundenheit mit dem Beruf

\begin{tabular}{lcccccc} 
& \multicolumn{3}{c}{$\begin{array}{c}\text { Zufriedenheit } \\
\text { mit der Ausbildung } \\
\text { (N=580) }\end{array}$} & \multicolumn{3}{c}{$\begin{array}{c}\text { Verbundenheit } \\
\text { mit dem Beruf } \\
\text { (N=570) }\end{array}$} \\
Sozioökonomischer Status & B & SE(B) & $\beta$ & $\mathrm{B}$ & $\mathrm{SE}(\mathrm{B})$ & $\beta$ \\
Migrationshintergrund & .00 & .00 & -.01 & -.01 & .00 & $-.09^{*}$ \\
Geschlecht & .01 & .03 & .01 & -.03 & .02 & -.05 \\
Schulische Herkunft & .06 & .09 & .02 & .10 & .06 & .06 \\
Selbstwert & .23 & .11 & $.08^{*}$ & .06 & .06 & .03 \\
Depressivität & .20 & .08 & $.11^{*}$ & .07 & .05 & .06 \\
Schulische Leistungsbereit. & .08 & .06 & .06 & .01 & .03 & .01 \\
Vielseitigkeit Betrieb & .07 & .08 & .04 & .10 & .05 & $.10^{*}$ \\
Handlungsspielraum Betrieb & .17 & .08 & $.10^{*}$ & .16 & .05 & $.17^{* *}$ \\
Klima Betrieb & .07 & .06 & .05 & .09 & .04 & $.12^{* *}$ \\
Belastung Betrieb & .36 & .07 & $.25^{* * *}$ & .20 & .04 & $.23^{* * *}$ \\
Vielseitigkeit Schule & .13 & .07 & $-.08^{*}$ & -.04 & .04 & -.04 \\
Handlungsspielraum Schule & .33 & .08 & $.19^{* * *}$ & .05 & .05 & .05 \\
Klima Schule & .02 & .06 & .01 & .06 & .03 & .06 \\
Belastung Schule & .07 & .07 & .04 & .08 & .04 & .08 \\
& -.09 & .07 & -.06 & .05 & .04 & .06
\end{tabular}

Anmerkungen. Zufriedenheit mit der Ausbildung $\mathrm{R}^{2}=.28, \mathrm{~F}=13.94(\mathrm{df}=16, \mathrm{p}<0.001)$, Verbundenheit mit dem Beruf $\mathrm{R}^{2}=.32, \mathrm{~F}=16.11(\mathrm{df}=16, \mathrm{p}<0.001)$

${ }^{* * *}=\mathrm{p}<.001,{ }^{* *}=\mathrm{p}<.01,{ }^{*}=\mathrm{p}<.05$

Die schulische Herkunft als Humankapital-Variable hängt lediglich mit der Ausbildungszufriedenheit zusammen: Sonderpädagogisch Beschulte sind eher zufriedener mit ihrer Ausbildungssituation als Lernende aus Regelklassen.

Dagegen spielen die hier erfassten stabilen Persönlichkeitsmerkmale bei allen Dimensionen des subjektiven Laufbahnerfolgs eine Rolle. Besonders wichtig ist ein hohes Selbstwertgefïhl, das positiv mit drei der vier abhängigen Variablen verknüpft ist: Am höchsten ist der Zusammenhang mit den selbsteingeschätzten Leistungen im Betrieb, einzig bei der beruflichen Verbundenheit ist er nicht signifikant. Depressivität zeigt einen erwartet negativen Zusammenhang, allerdings nur mit den selbsteingeschätzten betrieblichen Leistungen. Die schulische Leistungsbereitschaft schliesslich hängt nicht überraschend am deutlichsten mit der Selbsteinschätzung der schulischen Leistungen zusammen; etwas geringer auch mit der betrieblichen Selbsteinschätzung und der Ausbildungszufriedenheit.

Wie erwartet stehen die verschiedenen Einzelaspekte der Ausbildungsbedingungen in einem Zusammenhang mit subjektivem Laufbahnerfolg, wobei 
sich zeigt, dass der jeweilige Lernort für die entsprechenden Einschätzungen relevant ist (Fragestellung 2): Bei der schulischen Selbsteinschätzung spielen schulische Aspekte eine Rolle, bei der betrieblichen Selbsteinschätzung sind es dagegen erwartungsgemäss betriebliche Merkmale. Sowohl schulische wie noch ausgeprägter betriebliche Merkmale hängen mit der Ausbildungszufriedenheit zusammen, während dies bei der beruflichen Verbundenheit ausschliesslich für die betrieblichen Bedingungen gilt. Auch der Ausbildungstyp ist relevant, indem EBA-Lernende - unter Kontrolle der anderen Variablen - zufriedener sind als Anlehrlinge. Sie beurteilen allerdings ihre berufliche Verbundenheit tiefer und schätzen ihre betrieblichen Leistungen kritischer ein.

\section{Unterschiedliche Zusammenhänge je nach untersuchtem Kriterium subjektiven Laufbahnerfolgs?}

Es stellt sich nun umgekehrt die Frage, ob sich je nach untersuchtem Kriterium des subjektiven Laufbahnerfolgs unterschiedliche Zusammenhänge mit den unabhängigen Variablen zeigen (Fragestellung 3, vgl. Tabellen 4 und 5).

Die selbsteingeschätzten Leistungen in der Berufsfachschule (Fachkunde und Allgemeinbildung) stehen in einem Zusammenhang mit verschiedenen schulnahen Ausbildungsfaktoren und Persönlichkeitsmerkmalen: Die eigene schulische Leistungsbereitschaft, das Fehlen von schulischen Belastungsmomenten und ein positives Schulklima sind wichtig. Zudem ist ein positives Selbstwertgefühl mit günstigen Leistungseinschätzungen verknüpft. Die selbsteingeschätzte Leistung im Betrieb hängt dagegen mit verschiedenen betriebsnahen Faktoren, wie Handlungsspielraum und vielfältige Arbeit im Betrieb, zusammen. Weiter spielen persönliche Dispositionen eine Rolle. Aber auch sozio-demographische Faktoren (Geschlecht) und der Ausbildungstyp sind bedeutsam.

Den stärksten Zusammenhang mit der Ausbildungszufriedenheit haben verschiedene Ausbildungsaspekte (Betriebsklima, Vielseitigkeit in Schule und Betrieb, Ausbildungstyp). Zudem sind das Selbstwertgefühl und die schulische Herkunft bedeutsam: Sonderpädagogisch Beschulte sind eher zufriedener mit ihrer Ausbildungssituation als Lernende aus Regelklassen. Dagegen ist keiner der Faktoren auf der sozio-demographischen Ebene von Bedeutung.

Bei der beruflichen Verbundenheit zeigt sich ein ähnliches Bild wie bei der Ausbildungszufriedenheit, indem wiederum verschiedene Ausbildungsaspekte (Betriebsklima, vielseitige und autonome Arbeit) und der Ausbildungstyp am engsten damit gekoppelt sind. Zudem zeigt sich ein positiver Zusammenhang mit der schulischen Leistungsbereitschaft. Dagegen spielen wie schon oben die sozio-demographischen Faktoren keine wesentliche Rolle (Ausnahme: negativer Zusammenhang mit dem sozio-ökonomischen Status).

Dass Laufbahnerfolg ein durchaus facettenreiches Konzept mit unterschiedlichen Aspekten ist, verdeutlichen die relativ geringen Interkorrelationen zwischen den abhängigen Variablen; nur Ausbildungszufriedenheit und berufliche Verbundenheit korrelieren mit $\mathrm{r}=.45$ ( $\mathrm{p}<0.001)$ substanziell (Tab. 3$)$. 
Vergleicht man die Regressionskoeffizienten mit den bivariaten Korrelationen Tab. 3 fällt erwartungsgemäss auf, dass die Bedeutung bestimmter Variablen (insbesondere der Ausbildungsbedingungen in Schule und Betrieb, die untereinander korrelieren) unter Kontrolle der jeweils anderen Variablen in der Regressionsanalyse geringer wird oder ganz verschwindet.

\section{Diskussion der Ergebnisse}

MitdemZiel, den Forschungsstand überleistungsschwächere jungeErwachseneim schweizerischen Berufsbildungssystem zu erweitern, wurden zentrale Forschungsergebnisse zum subjektiven Laufbahnerfolg auf diese Gruppe übertragen. Dazu haben wir drei Fragen formuliert, die sich wie folgt beantworten lassen: Subjektiver Laufbahnerfolg hängt vor allem mit stabilen Persönlichkeitsmerkmalen und Arbeits- und Ausbildungsmerkmalen zusammen, während soziodemographische Merkmale und die schulische Herkunft (Humankapital) wenig relevant sind (Fragestellung 1). Die Unterscheidung zwischen betrieblichen und schulischen Einflussgrössen erwies sich in diesem Zusammenhang als relevant (Fragestellung 2) und die einzelnen Komponenten, die für die untersuchte Gruppe als Indikatoren für den subjektiven Laufbahnerfolg vorgeschlagen wurden, werden durch unterschiedliche Determinanten beeinflusst (Fragestellung 3).

$\mathrm{Ng}$ et al. (2005) argumentieren, dass Persönlichkeitsmerkmale eng mit affektiven Reaktionen auf die Arbeits- und Karrieresituation gekoppelt sind, somit sowohl die Selbstwahrnehmung wie auch die subjektive Wahrnehmung des eigenen Laufbahnerfolgs entscheidend beeinflussen. Da die Ergebnisse auf einem Querschnittsdesign beruhen, sind Schlussfolgerungen zu Ursache-Wirkungsbeziehungen hier allerdings problematisch: Führt eine positive Selbstwahrnehmung dazu, die eigenen Leistungen in einem besseren Licht zu betrachten, sie vielleicht auch eher sich selbst und nicht den Umständen zuzuschreiben? Plausibel wäre allerdings ebenfalls die Annahme, dass die aktuelle berufliche Situation in dieser kritischen Übergangsphase einen Einfluss auf ein ansonsten stabiles Selbstkonzept ausüben kann (Neuenschwander et al (2007)), v.a. da die Leistungen der Lernenden zum Befragungszeitpunkt kurz vor den Abschlussprüfungen besonders im Fokus standen. Diese Einschränkungen für die Interpretation aufgrund des Querschnittdesigns gelten natürlich für alle hier untersuchten Zusammenhänge.

Die betrieblichen Ausbildungsbedingungen sind für die Selbsteinschätzung der Leistungen ebenfalls zentral: Handlungsspielraum und Vielseitigkeit der Arbeit begünstigen eine positive Selbstwahrnehmung. Beides lässt sich auch als Angebot verstehen, die eigenen Fähigkeiten zu trainieren und weiterzuentwickeln und vom betroffenen Lernenden kann dies auch als Zeichen des Vertrauens in seine Fähigkeiten gedeutet werden. Entsprechend findet sich bei $\mathrm{Ng}$ et al. (2005) auch ein starker Zusammenhang zwischen «training and skill 
development opportunities» und subjektivem Laufbahnerfolg. Auch in der Studie von Lehmann und Seeber (2007) versicherten die Auszubildenden retrospektiv, dass sie ihr fachliches Wissen und Können vor allem dank betrieblicher Ausbildungsanteile erworben haben. In unserer Studie sind im schulischen Umfeld andere Einflussfaktoren (Klima, wahrgenommene Belastung) entscheidender für die Leistungseinschätzung. Vermutlich lässt sich dies auch damit erklären, dass der schulische Anteil stärker standardisiert und die Varianz v.a. in Bezug auf die Variable "Handlungsspielraum» geringer ist als im betrieblichen Umfeld.

Auch die berufliche Verbundenheit und die Ausbildungszufriedenheit werden eher durch betriebliche als durch schulische Faktoren beeinflusst. Amos et al. (2003) fanden hinsichtlich der Ausbildungszufriedenheit bei den betrieblichen Ausbildungsmerkmalen ebenfalls einen grossen Anteil der aufgeklärten Varianz (36\%), der durch schulische Faktoren nur noch um 1\% erhöht werden könnte. Diese Ergebnisse sind angesichts des unterschiedlich hohen zeitlichen Anteils der beiden Lernorte nachvollziehbar (80\% im Betrieb, $20 \%$ in der Berufsfachschule). Allerdings sind im Vergleich mit der Leistungseinschätzung hier andere betriebliche Merkmale entscheidend, insbesondere das Betriebsklima, das für die Leistungseinschätzung vollkommen unbedeutend war. Zusammenfassend lässt sich festhalten, dass sich die differenzierte Erfassung der Ausbildungsvariablen als sinnvoll erweist, sowohl im Hinblick auf die Unterscheidung der beiden Lernorte als auch auf die Erfassung unterschiedlicher Merkmale. Aus methodischer Sicht ist hier allerdings zu ergänzen, dass die hohen Interkorrelationen bei der Interpretation zu berücksichtigen sind und die hohe Zahl der Prädiktoren die Wahrscheinlichkeit von Zufallssignifikanzen erhöht. Aus praktischer Sicht weisen die Resultate darauf hin, dass subjektiver Laufbahnerfolg von der betrieblichen Seite her aktiv beeinflusst werden kann und die Betriebe hier einiges an Verantwortung tragen, indem sie den Lernenden bestimmte Erfahrungen ermöglichen und sie begleiten.

Interessant ist, dass sich die sozio-demographischen Faktoren und die schulische Herkunft zwar insgesamt nicht bedeutend, aber wenn, dann eher nicht erwartungsgemäss auswirken: Man hätte annehmen können, dass Jugendliche mit Migrationshintergrund, aus tieferen sozialen Schichten und Jugendliche aus Sonderschulen oder -klassen ihren subjektiven Laufbahnerfolg eher kritischer einschätzen (Müller, 2009). Dies ist jedoch in unserer Gruppe nicht der Fall: Migrationsjugendliche beurteilen ihre betrieblichen Leistungen positiver, Jugendliche aus tieferen sozialen Schichten ihre berufliche Verbundenheit höher und Lernende, die ehemals Sonderschulen oder Sonderklassen besuchten, sind insgesamt mit der Ausbildung zufriedener. Eine mögliche Erklärung ist der einleitend erwähnte Umstand, dass diese Personengruppen gemessen an ihrer jeweiligen sozialen/schulischen Referenzgruppe, einen relativ positiven Berufseinstieg zu verzeichnen haben (Heslin, 2005).

Vor dem Hintergrund der Berufsbildungsreform wäre es natürlich interessant zu analysieren, ob sich der Einfluss bestimmter Faktoren in der neuen Situation 
verändert hat, da befürchtet wurde, dass gewisse Jugendliche mit den neuen erhöhten Anforderungen überfordert sein könnten. Zusätzliche Analysen der Subgruppen (Anlehre-EBA), die mangels Vergleichsdaten nur in der Gastronomie und im Detailhandel durchgeführt werden konnten, ergaben allerdings nur marginal andere Regresssionskoeffizienten und insgesamt kein klar $\mathrm{zu}$ interpretierendes Bild. Feststellen lässt sich aber, dass sich die neue Ausbildungsform nicht negativ auf die schulische Leistungseinschätzung auswirkt und die EBA-Lernenden mit der Ausbildung zufriedener sind. Etwas kritischer als die Anlehrlinge beurteilen sie dagegen ihre betrieblichen Leistungen. Die geringere berufliche Verbundenheit der EBA-Lernenden ist zweischneidig: Junge Berufsleute, die sich mit ihrem Beruf wenig verbunden fühlen, wechseln vielleicht eher das Berufsfeld und es fragt sich, ob sie so ihre Kompetenzen optimal nutzen und sich ihre Investition und die des Betriebs gelohnt hat. Auf der anderen Seite ist die tiefere berufliche Verbundenheit vielleicht auch ein Ausdruck davon, dass sie ihre Chancen, sich beruflich neu zu orientieren und mobil zu sein, erkennen und die "Durchlässigkeit» so in einem erweiterten Sinn stattfindet. $\mathrm{Zu}$ ergänzen ist, dass die Selektion der Lehrbetriebe vor der Ausbildung und die Anforderungen der Ausbildung selber dazu beigetragen haben dürften, dass die am Ausbildungsende befragten Jugendlichen, die "durchgehalten» haben, tendenziell zu den Leistungsstärkeren gehören. So beurteilen die Lehrpersonen die Situation denn auch insgesamt klar kritischer (Hofmann \& Kammermann, 2008) und beziehen sich dabei vermutlich auf ihre allgemeine Erfahrung mit ihren bisherigen Lernenden (inkl. der «drop-outs»).

Abschliessend stellt sich die Frage, wie man diese subjektiven Faktoren aus bildungs- und gesellschaftspolitischer Sicht gewichtet, da man auch nach wie vor davon ausgehen muss, dass soziale Benachteiligung in ihren verschiedenen Facetten den objektiven Laufbahnerfolg negativ beeinflusst (Hupka, Sacchi \& Stalder, 2010). Wohlbefinden und Zufriedenheit wurden in einem anderen Zusammenhang als wichtige Determinanten für erfolgreiches schulisches Lernen genannt (Neuenschwander \& Hascher, 2003). So lässt sich zumindest vermuten und hoffen, dass junge Berufsleute, die das Ausbildungssystem zufrieden und mit Erfolgserlebnissen verlassen, ihre Laufbahn motiviert angehen und so die Chancen gut stehen, dass der subjektive wahrgenommene Laufbahnerfolg sich in objektiven Erfolg umsetzen lässt. Zusätzliche Analysen der vorhandenen Daten, die auch die Nachbefragungen jeweils ein Jahr nach Ausbildungsabschluss miteinbeziehen, werden in dieser Hinsicht weitere interessante Erkenntnisse ermöglichen.

\section{Anmerkung}

1 Folgende Variablen haben besonders hohe Interkorrelationen ( $r>4)$, die bei der Interpretation zu berücksichtigen sind: Klima Schule - Leistungsbereitschaft Schule ( $r=.46)$, Vielseitigkeit Schule - Leistungsbereitschaft Schule ( $\mathrm{r}=.57)$, Klima Betrieb - Vielseitigkeit Betrieb ( $\mathrm{r}=.54)$, Klima Betrieb - Handlungsspielraum Betrieb ( $\mathrm{r}=.46)$, Klima Schule Vielseitigkeit Schule ( $r=.49)$, Vielseitigkeit Betrieb - Handlungsspielraum Betrieb ( $r=.53)$ 


\section{Literatur}

Baillod, J. (1992). Fluktuation bei Computerfachleuten. Eine Längsschnittuntersuchung über die Beziehungen zwischen Arbeitssituation und Berufsverläufen. Bern: Peter Lang.

Becker, R. \& Hecken, A. E. (2008). Why are working-class children diverted from universities? - An empirical assessment of the diversion thesis. European Sociological Review, 25 (2), 233-250.

Bundesamt für Berufsbildung und Technologie (BBT). (2005). Leitfaden zur zweijährigen beruflichen Grundbildung. Bern: BBT.

Elfering, A., Semmer, N., Tschan, F., Kälin, W. \& Bucher, A. (2007). First years in job: A three-wave analysis of work experiences. Journal of Vocational Behavior, 70, 97-115.

Field, A. (2009). Discovering statistics using SPSS (3rd ed.). London: SAGE.

Hackman, J. R. \& Oldham, G. R. (1976). Motivation through the design of work: Test of a theory. Organizational Behavior and Human Performance, 16, 250-279.

Häfeli, K. \& Schellenberg, C. (2009). Erfolgsfaktoren in der Berufsausbildung bei gefährdeten Jugendlichen. Bern: EDK (Schweizerische Konferenz der kantonalen Erziehungsdirektoren).

Herzog, W., Neuenschwander, M. P. \& Wannack, E. (2006). Berufswahlprozess. Wie sich Jugendliche auf ihren Beruf vorbereiten. Bern: Haupt.

Heslin, P. A. (2005). Conceptualizing and evaluating career success. Journal of Organizational Behavior, 26, 113-136.

Hofmann, C. \& Kammermann, M. (2008). Die zweijährige berufliche Grundbildung aus Sicht der Ausbildungsverantwortlichen in Berufsfachschule und Betrieb. Schweizerische Zeitschrift für Heilpädagogik, 6, 43-50.

Hofmann, C. \& Kammermann, M. (2009). Die zweijährige berufliche Grundbildung - ein Erfolgsmodell? Schweizerische Zeitschrift für Heilpädagogik, 6, 27-34.

Humphrey, S. E., Nahrgang, J. D. \& Morgeson, F. P. (2007). Integrating motivational, social, and contextual work design features: A meta-analytic summary and theoretical extension of the work design literature. Journal of Applied Psychology, 92 (5), 1332-1356.

Hupka, S., Sacchi, S. \& Stalder, B. E. (2010). Social origin and access to upper secondary education in Switzerland: A comparison of company-based apprenticeship and exclusively school-based programmes. Swiss Journal of Sociology, 36 (1), 11-31.

Kälin, W., Semmer, N. K., Elfering, A., Tschan, F., Dauwalder, J.-P., Heunert, S. \& Crettaz von Roten, F. (2000). Work characteristics and well-being of Swiss apprentices entering the labor market. Swiss Journal of Psychology, 59 (4), 272-290.

Kammermann, M. (2010). Job or further training? Education and Training, 52 (5), 391-403.

Kammermann, M., Amos, J., Hofmann, C. \& Hättich, A. (2009). Integriert in den Arbeitsmarkt? Personen mit Berufsattest im Detailhandel und im Gastgewerbe ein Jahr nach Ausbildungsabschluss. Zürich: Hochschule für Heilpädagogik.

Kammermann, M. \& Hättich, A. (2010). Mit Berufsattest in den Arbeitsmarkt. Berufsbildung in Wissenschaft und Praxis, 5, 11-14.

Kammermann, M., Hofmann, C. \& Hättich, A. (2009). Forschungsprojekt Arbeitsmarktfähigkeit und zweijährige berufliche Grundbildung. Skalendokumentation zur Ersterhebung bei den Lernenden. Zürich: Hochschule für Heilpädagogik.

Lehmann, R. \& Seeber, S. (Hrsg.). (2007). ULME III. Untersuchung von Leistungen, Motivation und Einstellungen der Schülerinnen und Schüler in den Abschlussklassen der Berufsschulen. Hamburg: Hamburger Institut für berufliche Bildung (HIBB).

Moser, U. (1997). Messinstrumente zu Unterricht und Leistungsbereitschft in TIMSS +. Projektdokumentation. Bern: Amt für Bildungsforschung der Erziehungsdirektion.

Moser, U., Ramseier, E., Keller, C. \& Huber, M. (1997). Schule auf dem Prüfstand. Eine 
Evaluation der Sekundarstufe I auf der Grundlage der "Third International Mathematics and Science Study». Chur: Rüegger.

Müller, R. (2009). Berufswahl und Lehre. Berufliche Orientierungs- und Entscheidungsprozesse bei ausländischen und schweizerischen Jugendlichen (1. Auflage). Bern: hep-Verlag.

Myers, R. (1990). Classical and mordern regression with applications (2nd ed.). Boston, MA: Duxbury.

Neuenschwander, M. P. (1998). Schule und Identität im Jugendalter I. Kurzdokumentation der Skalen und Stichproben (Forschungsbericht Nr. 18). Universität Bern, Abteilung Pädagogische Psychologie.

Neuenschwander, M. P., Frey, M. \& Gasser, L. (2007). Übergang in die Sekundarstufe II und Wirkungen der Berufsbildung. Zürcher Ergänzungsstudie Berufsbildung - Schlussbericht. Jacobs Center for Productive Youth Development, Universität Zürich.

Neuenschwander, M. P., Gerber, M., Frank, N. \& Rottermann, B. (Hrsg.). (2012). Schule und Beruf. Wege in die Erwerbstätigkeit. Wiesbaden: VS Verlag.

Neuenschwander, M. P. \& Hascher, T. (2003). Zufriedenheit von Schülerinnen und Schülern und ihre soziale Integration. Psychologie in Erziehung und Unterricht, 50, 270-280.

Ng, T. W. H., Eby, L. T., Sorensen, K. L. \& Feldman, D. C. (2005). Predictors of objective and subjective career success: A meta-analysis. Personnel Psychology, 58, 367-408.

OECD. (2011). Bildung auf einen Blick. OECD-Indikatoren 2011. Paris: OECD.

Prümper, J., Hartmannsgruber, K. \& Frese, M. (1995). KFZA. Kurz-Fragebogen zur Arbeitsanalyse. Zeitschrift für Arbeits- und Organisationspsychologie, 39 (3), 125-131.

Rosenberg, M. (1979). Conceiving the self. New York: Basic Books.

Schmid, E. \& Stalder, B. E. (2008). Lehrvertragsauflösung: Chancen und Risiken für den weiteren Ausbildungsweg. Ergebnisse aus dem Projekt LEVA. Bildungsplanung und Evaluation der Erziehungsdirektion des Kantons Bern.

Semmer, N., Zapf, D. \& Dunckel, H. (1999). Instrument zur Stressbezogenen Tätigkeitsanalyse (ISTA). In H. Dunckel (Hrsg.), Handbuch psychologischer Arbeitsanalyseverfahren (S. 179-205). Zürich: vdf Hochschulverlag an der ETH.

Stalder, B. E. (2003). Schule, Arbeit und Ausbildungszufriedenheit. In BFS \& TREE (Hrsg.), Wege in die nachobligatorische Ausbildung (S. 59-80). Neuenburg: Bundesamt für Statistik.

Stalder, B. E., Meyer, T. \& Hupka-Brunner, S. (2011). TREE Project documentation. In M. M. Bergman, S. Hupka-Brunner, A. Keller, T. Meyer \& B. E. Stalder (Hrsg.), Youth Transitions in Switzerland: Results from the TREE panel study (pp. 66-87). Zürich: Seismo.

Schlagworte: Berufsbildung, Laufbahnerfolg, Jugendliche, Leistungsschwächere 


\title{
Le succès subjectif du parcours professionnel des jeunes avec de faibles performances
}

\begin{abstract}
Ré sumé
L'entrée dans la vie active est rendue difficile pour des jeunes à faibles performances. Selon des critères objectifs (salaire, position), ils réussissent la plupart du temps moins bien professionnellement. Qu'en est-il cependant de l'auto-appréciation subjective quant au succès du parcours professionnel propre en début de carrière et quels facteurs l'influencent? 525 apprentis en formation professionnelle initiale avec attestation fédérale de formation professionnelle (AFP) de quatre branches, ainsi que 183 apprentis en fin de formation ont été sondés dans le cadre d'une étude (2005-2011): les résultats montrent que le succès subjectif est principalement est lié à des critères de personnalité, de travail et de formation, bien que ce soit principalement la situation de formation en entreprise qui soit significative. Les critères sociodémographiques et l'origine scolaire par contre ne ressortent que de manière minime.
\end{abstract}

Mots clés: Formation professionelle, succès professionelle, jeunes, difficultés d'apprentissage

\section{Formazione professionale per giovani dalle prestazioni deboli: valutazione del successo da un punto di vista soggettivo}

\section{Riassunto}

Per giovani dalle prestazioni deboli è difficile iniziare un'attività lavorativa. Stando a criteri oggettivi (salario, posizione) hanno generalmente meno successo professionale. $\mathrm{Ci}$ si può però anche chiedere come loro valutino da un punto di vista soggettivo il proprio successo all'inizio della carriera professionale, e da quali fattori tale carriera venga condizionata. In una ricerca (2006-2011) sono stati intervistati alla fine dei corsi 525 apprendisti in formazione di base con certificato federale di formazione pratica (CFP) in quattro rami differenti, come pure 183 giovani che frequentavano un apprendistato pratico. Stando ai risultati, la visione soggettiva del successo viene influenzata da fattori legati sia alla personalità, sia al lavoro e alla formazione. Di particolare importanza risultano gli aspetti aziendali che hanno a che fare con la situazione formativa. Pertanto le caratteristiche sociodemografiche e il passato scolastico hanno un effetto minore.

Parole chiave: Formazione professionale, successo professionale, giovani, difficoltà di apprendimento 


\title{
Subjective career success of young people with learning disabilities in VET
}

\begin{abstract}
For young people with learning disabilities career start is often difficult. As measured by objective criteria (salary, position) they are also less successful in their professional life. But what's about their subjective view of career success at the beginning of their career and which factors are influencing it? In a study (2006-2011) 525 trainees in a basic training with Federal VET Certificate and 183 trainees of a VET elementary training program of four branches were questioned at the end of their training. Results show that subjective career success is particularly related to personality, work features and training conditions, especially those at the company. Socio-demographic characteristics and school background are less important.
\end{abstract}

Key words: Vocational education and training, career success, youth, learning disabilities 
